\title{
Intratumoral Injection of a Human Papillomavirus Therapeutic Vaccine-Induced Strong Anti-TC- I-Grafted Tumor Activity in Mice
}

\author{
Yuxin Che \\ Yang Yang' \\ Jinguo Suo' \\ Chang Chen ${ }^{2}$ \\ Xuelian Wang (D) \\ 'Department of Microbiology and \\ Parasitology, College of Basic Medical \\ Science, China Medical University, \\ Shenyang, Liaoning, People's Republic of \\ China; ${ }^{2}$ Department of Dermatology, The \\ First Hospital of China Medical \\ University, Shenyang, Liaoning, People's \\ Republic of China
}

Purpose: The route of administration of a therapeutic tumor vaccine is a critical factor in inducing antitumor activity. In this study, we explored the effects of three vaccination routes (subcutaneous, peritumoral, and intratumoral injection) on antitumor activity induced by a human papillomavirus (HPV) therapeutic vaccine containing HPV16 E7 peptide combined with the adjuvant $\mathrm{CpG}$ ODN in established TC-1 grafted tumors.

Methods: We used flow cytometry to evaluate splenic and tumor-infiltrating immune cells. We also assessed transcriptional changes in a sequence of immune-related genes in tumors of different treatment groups using quantitative real-time polymerase chain reaction. Immunohistochemistry was used to determine the expression of molecules related to tumor infiltrating immune cells, angiogenesis, and cancer-associated fibroblasts in tumor tissues.

Results: Our results suggested that intratumoral and peritumoral vaccination generated enhanced antitumor activity compared to subcutaneous delivery. In particular, intratumoral vaccination elicited a stronger antitumor effect, with two of the six treated mice being nearly tumor-free at day 28. Three vaccination routes induced increases in splenic CD4+ and/or CD8+ T lymphocytes, and marked decreases in immunosuppressive cells. Peritumoral vaccination increased the tumor-infiltrating $\mathrm{CD} 8+\mathrm{T}$ cells in tumors, while intratumoral vaccination enhanced the tumor-infiltrating $\mathrm{CD} 4+$ and $\mathrm{CD} 8+\mathrm{T}$ lymphocytes, as well as decreased the tumor-infiltrating of immunosuppressive cells, which may result in stronger inhibition of tumor growth and prolonged survival in mice bearing tumors. Furthermore, compared to the subcutaneous route, intratumoral vaccination led to a significant increase in antitumor cytokines and chemokines. In addition, our data showed marked downregulation of MMP-2, MMP-9, VEGF, CD31, and $\alpha$-SMA in the intratumoral vaccination group, which might contribute to the suppression of tumor invasion, angiogenesis, and metastasis.

Conclusion: Overall, intratumoral vaccination is superior to subcutaneous delivery and has the potential to inhibit tumor growth by improving the tumor microenvironment.

Keywords: human papillomavirus, cervical cancer, therapeutic vaccine, intratumoral injection, immunosuppression

\section{Introduction}

Human papillomavirus (HPV) infection is the most common sexually transmitted infection worldwide and persistent infection with high-risk HPV (especially type 16 and 18) can cause cancer of the cervix, vulva, vagina, anus, penis, and oropharynx. ${ }^{1}$ The enormous global health burden of high-risk HPV persistent infections has promoted extensive prophylactic vaccine development, which have primarily aimed to induce the generation of neutralizing antibodies against the HPV L1
Department of Microbiology and

Parasitology, College of Basic Medical

Science, China Medical University, No. 77

Puhe Road, Shenyang North New Area,

Shenyang, I I0122, People's Republic of

China

Tel/Fax +86 24-31939077

Email xlwang18@cmu.edu.cn 
capsid protein. ${ }^{2}$ Though immune responses induced by the prophylactic vaccines confer protection against infection due to the HPV genotypes included in the vaccines, these prophylactic vaccines have no therapeutic effect on either pre-existing infections or established HPV-associated diseases. $^{3}$

Cell-mediated immune responses involving T-helper type 1 (Th1) cells and cytotoxic $\mathrm{T}$ cells are important for the clearance of established HPV-related diseases. HPVassociated malignancies are driven by HPV oncoproteins E6 and E7, which are required for cellular malignant transformation and are expressed continuously at high levels in transformed cells, and act as tumor-specific antigenic targets. ${ }^{4}$ An ideal therapeutic vaccine for high-risk HPV-induced cancers could target these oncoproteins to induce robust oncoproteins-specific Th1 and cytotoxic $\mathrm{T}$ lymphocyte (CTL) responses that kill HPV-infected and HPV-transformed cells. ${ }^{5}$ Our prior study found that subcutaneous injection of a vaccine consisting of the HPV16 E7 43-77 peptide, which contains both a CTL epitope (E7 49-57) and two Th epitopes (E7 50-62 and E7 43-77), and the adjuvant, unmethylated cytosinephosphate-guanosine oligodeoxynucleotide ( $\mathrm{CpG}$ ODN), induced strong antitumor immune responses and resulted in the eradication of early stage implanted tumors. ${ }^{6,7}$

A successful cancer therapeutic vaccine should be able to induce a powerful immune response against tumorspecific-antigens to avoid targeting normal host cells and overcome immunosuppression. ${ }^{8}$ Vaccine immunogenicity may be affected by how the antigens are presented, as well as the vaccine dose, and the administration route of vaccine. ${ }^{9}$ Conventional vaccines are often administered to the skin or muscle to activate $\mathrm{T}$ cells in lymph nodes. Direct intratumoral delivery achieves high local concentration of the vaccine and increases the bioavailability of immunostimulatory products. ${ }^{10}$ A number of studies have shown that cancer therapeutic vaccines might be more immunogenic when delivered intratumorally. ${ }^{11-18}$ Instead, other studies have suggested that peritumoral injection of vaccines resulted in eradication or strong growth inhibition of established tumors. ${ }^{19,20}$ In HPV-related skin and mucosal tumors, peritumoral injection and intratumoral injection of vaccines are feasible. ${ }^{21}$ Building on these observations, we extended our study of vaccines to TC1-grafted tumors, and compared the antitumor efficacy induced by three different routes (subcutaneous injection, peritumoral injection, and intratumoral injection) of the vaccine. The results showed that intratumoral vaccination was far superior to the subcutaneous route at inducing the inhibition of tumor growth and improvement of the tumor microenvironment (TME), which may provide a significant benefit for the treatment of HPV-associated diseases.

\section{Materials and Methods Mouse Model and Cell Line}

Female C57BL/ 6 mice ( 6 to 8 weeks of age) were obtained from the Liaoning Changsheng Biotechnology Co. Ltd. (Benxi, China) and housed in a specific pathogen-free environment. All animal procedures were performed in accordance with the Guidelines for the Care and Use of Laboratory Animals and were approved by the Institutional Animal Care and Use Committee of China Medical University (IACUC Issue No. CMU2019293).

The TC-1 cell line was purchased from Beijing Beina Chuanglian Biotechnology Institute (Beijing, China). TC-1 cells were cultured in Roswell Park Memorial Institute 1640 medium supplemented with G418 (Genview, Tallahassee, FL, USA), 10\% heat-inactivated fetal bovine serum, and penicillin-streptomycin (all from Biological Industries, Kibbutz Beit Haemek, Israel).

\section{Peptide and Adjuvant Treatments}

The HPV16 E7 peptide (E7 43-77: GQAEPDRAH YNIVTFCCKCDSTLRLCVQSTHVDIR) was provided by GL Biochem (Shanghai) Ltd. (Shanghai, China). The purity of the peptide was verified by high-performance liquid chromatography and was found to be routinely $>95 \%$. CpG ODN 1826 (5'-TCCATGACGTTCCTGACGTT-3') was synthesized by Sangon Biotech (Shanghai) Co. Ltd. (Shanghai, China).

\section{Tumor Treatment and Monitoring}

C57BL/6 mice (6 mice per group) were subcutaneously inoculated with $5 \times 10^{5} \mathrm{TC}-1$ tumor cells at day 0 . Tumorbearing mice were randomly treated with a vaccine containing $20 \mu \mathrm{g}$ of $\mathrm{CpG}$ and $50 \mu \mathrm{g}$ of $\mathrm{E} 7$ peptide in a total volume of $100 \mu \mathrm{L}$ by subcutaneous injection, peritumoral injection, or intratumoral injection. Tumor volumes were measured using an electronic caliper (Pro'skit, Shanghai, China) and calculation of tumor dimensions were performed using the formula $\mathrm{V}=0.5 \times \mathrm{a} \times \mathrm{b}^{2}$, where a was the longest superficial diameter and $\mathrm{b}$ was the smallest one. To determine the overall survival of the treated tumor-bearing mice, we euthanized mice and counted a death event when 
the tumor volume of the mouse reached $2000 \mathrm{~mm}^{3}$ or when the tumor diameter was greater than $1.5 \mathrm{~cm}$.

\section{Flow Cytometry}

The mice were euthanized, and the spleens and tumors were harvested at day 28. To prepare the splenocyte suspensions, mouse spleens were disrupted and passed through a stainless-steel mesh. Tumor single-cell suspensions were obtained by digesting dissected tumor tissues with $2 \mathrm{mg} / \mathrm{mL}$ Collagenase D (Roche, Diagnostics, Indianapolis, IN, USA) and $0.4 \mathrm{mg} / \mathrm{mL}$ DNase (SigmaAldrich, St. Louis, MO) for 90 minutes at $37^{\circ} \mathrm{C}$ and subsequently the tumor cells were filtered using a $100 \mu \mathrm{m}$ strainer.

For surface staining, single-cell suspensions were stained with the following fluorochrome-conjugated monoclonal antibodies: FITC-conjugated anti-mouse CD4 (BD Pharmingen, San Diego, CA, USA), PerCP/Cy5.5conjugated anti-mouse CD8a (BD Pharmingen, San Diego, CA, USA), PerCP/Cy5.5-conjugated anti-mouse CD11b (eBioscience, San Diego, CA, USA), APC-conjugated anti-mouse CD25 (BD Pharmingen, San Diego, CA, USA), FITC-conjugated anti-mouse Gr-1 (eBioscience, San Diego, CA, USA), and PE-conjugated anti-mouse F4/80 (eBioscience, San Diego, CA, USA).

For intracellular interferon gamma (IFN- $\gamma$ ) staining, cells were incubated with $1 \mu \mathrm{g} / \mathrm{mL}$ Phorbol 12-myristate 13-acetate and $50 \mu \mathrm{g} / \mathrm{mL}$ Ionomycin (all from SigmaAldrich, St. Louis, MO, USA). After the 1-hour incubation, GolgiPlug (BD Biosciences, San Diego, CA, USA) was added for 4 hours to inhibit protein secretion. Intracellular CD206 and IFN- $\gamma$ staining was performed using the Cytofix/Cytoperm kit (BD Biosciences, San Diego, CA, USA) according to the manufacturer's instructions. Following the fixation/permeabilization, cells were additionally stained with APC-conjugated anti-mouse CD206 (eBioscience, San Diego, CA, USA), PEconjugated anti-mouse IFN- $\gamma$ (BD Pharmingen, San Diego, CA, USA) or isotype controls.

For intranuclear staining of Foxp3, cells were fixed and permeabilized using the Foxp3/Transcription Factor Staining Buffer Set (Invitrogen, Carlsbad, CA, USA). Following the fixation and permeabilization, cells were stained with PE-conjugated anti-mouse Foxp3 (eBioscience, San Diego, CA, USA). Analysis was performed using a BD LSRFortessa flow cytometer (BD Biosciences, USA) and FlowJo software (TreeStar, Ashland, OR, USA).

\section{Immunohistochemistry}

Immunohistochemical staining was performed on formalin-fixed and paraffin-embedded tissue. Heat-induced antigen retrieval was performed with EDTA buffer at $\mathrm{pH}=9.0$. Sections (3- $\mu \mathrm{m}$ thick) were treated with the following primary antibodies: CD4, CD8, CD31, and alpha-smooth muscle actin ( $\alpha$-SMA) (rabbit polyclonal antibodies, ABclonal Biotech Co, Ltd, Wuhan, China, 1:200 dilution) and incubated overnight at $4^{\circ} \mathrm{C}$. After overnight incubation, the slides were treated with biotinylated secondary antibodies and streptavidin-horseradish peroxidase (Maixin Biotechnology, Fuzhou, Fujian, China). The final stain was developed using 3,3'-diaminobenzidine tetrahydrochloride (Maixin Biotechnology, Fuzhou, Fujian, China) as the chromogen. The stained sections were counterstained with hematoxylin and coverslipped.

Staining intensity was scored on a four-point scale: negative (no stain, 0), weak (1), moderate (2), and strong (3). The extent of staining (percentage of positive tumor cells) was scored as follows: $0(0 \%), 1(1 \%-10 \%), 2$ $(11 \%-50 \%), 3(51 \%-80 \%)$, and $4(>80 \%)$. The intensity scores and extent of staining were multiplied to give a composite score ranging from 1 to 12 for each tumor.

\section{RNA Extraction and Quantitative Real-Time Reverse Transcription Polymerase Chain Reaction}

Total RNA was extracted from whole tumors using TRIzol Reagent (Invitrogen, Carlsbad, CA, USA) according to the manufacturer's instructions. The extracted RNA was reverse transcribed using a Reverse Transcription System (Promega, Madison, WI, USA) for cDNA synthesis. PCR analysis was performed using the SYBR qPCR Master Mix (Vazyme, Nanjing, China). Specific primers used for the quantitative real-time reverse transcription polymerase chain reaction (qRT-PCR) experiments are listed in Table 1. $\beta$-actin was used as the endogenous control. The $2^{-\Delta \Delta \mathrm{Ct}}$ method was used to calculate the relative quantity of gene expression.

\section{Statistical Analysis}

All statistical analyses were performed using GraphPad Prism software version 5.0 (GraphPad Software Inc., San Diego, CA, USA). Survival analysis curves were created using Kaplan-Meier methods and compared by the Log rank test. The rest of data were analyzed using one-way analysis of variance (ANOVA) followed by Tukey's 
Table I qRT-PCR Primers Used in the Study

\begin{tabular}{|l|l|l|}
\hline Gene & \multicolumn{1}{|c|}{ Forward (5''-3') } & \multicolumn{1}{c|}{ Reverse (5'-3') } \\
\hline$\beta$-actin & CATCCGTAAAGACCTCTATGCCAAC & ATGGAGCCACCGATCCACA \\
IL-2 & AAGCTCTACAGCGGAAGCAC & TCATCGAATTGGCACTCAAA \\
IL- 12 & AGGATGCACATCAAAAGGCTT & GGCCTCGGTTAGGAAGGATAC \\
TNF- $\alpha$ & CAATCACGCTACCTCCTCTTTT & CAGCAGTGCAGGAATAATGTTTC \\
TGF- $\beta$ & CCAACATGCTGATTGATGACACC & GAGAATGCCAATTTTGATTGCCA \\
IFN- $\gamma$ & AATGGTACCGTCAGTGCTGGAAATA & TGGCTCATGTTGCAGAGGCTA \\
CXCLI & ACTCMGTGGCATAGATGTGGMG & GACGCTTATGTTGTTGCTGATGG \\
CXCL9 & CTGGGATTCACCTCMGAACATC & CAGGGTCAAGGCAAGCCTC \\
CXCLI0 & CTTGAGCCTAGTCGTGATAAC & CCAGCTTGGTGAGGTCTATC \\
MMP-2 2 & CCACGTGTTGAGATCATTGC & AGTAGCAGCTGATGTGACC \\
MMP- 9 & CATCGTAGTTGGCTGTGGTCG & GTCTTCCCCTTCACTTTCCTG \\
VEGF & GCAGAGGCATACTTGTACCG & TGATGTTATGATGGTCCCACTTG \\
\hline
\end{tabular}

multiple comparison test. Differences were considered significant at a $\mathrm{p}$-value $<0.05$. The data are presented as the mean \pm standard deviation (SD). P-values were as follows: $* \mathrm{p}<0.05, * * \mathrm{p}<0.01$, and $* * * \mathrm{p}<0.001$.

\section{Results}

\section{Peritumoral or Intratumoral Injection of Vaccine Induced Inhibition of Established Tumors}

To investigate a potential antitumor effect of different administration routes of vaccine, TC-1 tumor cells were injected subcutaneously in the right flank of mice on day 0 . When the tumor volume reached $150 \pm 50 \mathrm{~mm}^{3}$, mice were treated with vaccine containing $20 \mu \mathrm{g}$ of $\mathrm{CpG}$ and 50 $\mu \mathrm{g}$ of E7 peptide on days 9,16 , and 23 by subcutaneous injection, peritumoral injection or intratumoral injection (Figure 1A). Mice with TC-1 grafted tumors showed variation in the response to different administration routes of the vaccine. Tumors maintained sustained development in all mice in the control group. The majority of tumors in mice receiving subcutaneously injected vaccine grew slower than untreated mice. All mice treated with peritumoral or intratumoral injection of the vaccine responded almost completely or partially reduction in tumor volume (Figure 1B). The peritumoral or intratumoral injection of the vaccine significantly inhibited tumor growth, as indicated by $60.86 \%(\mathrm{p}<0.001)$ and $67.10 \%(\mathrm{p}<0.001)$ reductions, respectively, in mean tumor volume compared to that of control mice, as well as $53.85 \%(\mathrm{p}<0.01)$ and $61.21 \%(\mathrm{p}<0.001)$, respective reductions relative to subcutaneous injected mice (Figure 1C). At the endpoint of the experiment, tumors were excised from the mice and weighed (Figure 1D). The peritumoral $(1.64 \pm 0.63 \mathrm{~g})$ or intratumoral $(1.49 \pm 1.25 \mathrm{~g})$ injection of the vaccine resulted in significantly decreased in tumor weight compared to the control group $(4.43 \pm 2.14 \mathrm{~g}, \mathrm{p}<0.01)$, and the subcutaneous injected group $(3.77 \pm 0.56 \mathrm{~g}, \mathrm{p}<0.05)$ (Figure 1E). Figure $1 \mathrm{~F}$ presents the survival curves of mice treated with different administration routes of the vaccine. The first mice were found dead on day 14 after TC-1 cells were inoculated in the control group (Figure 1F). In vaccinated mice, a significant delay in time to death was registered. At 50 days after the inoculation of TC-1 cells, the percentage of mice that survived in the subcutaneous and peritumoral injected group was $33.33 \%$ and $50 \%$, respectively (Figure $1 \mathrm{~F}$ ). The percentage of animals that survived was $83.33 \%$ in the intratumoral injected group at the endpoint of the experiment (Figure 1F). In short, these data indicated that the peritumoral and intratumoral treatment showed significantly improved antitumor efficacy on the inhibition of tumor growth and prolonged mouse survival compared with the control group. Although there was no statistical difference between the peritumoral injection group and the intratumoral injection group, intratumoral vaccination achieved better effects on the inhibition of tumor growth and two of the six mice were nearly tumor-free at day 28 .

\section{Effects of Different Administration Routes of the Vaccine on Systemic Immune Responses}

To evaluate the effects of three different administration routes of the vaccine on the systemic immune responses, 
A

$\downarrow$ Subcutaneous injection $\quad \downarrow$ Peritumoral injection $\quad \downarrow$ Intratumoral injection

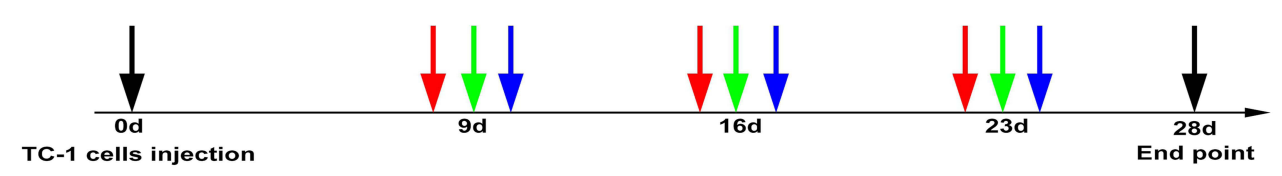

B
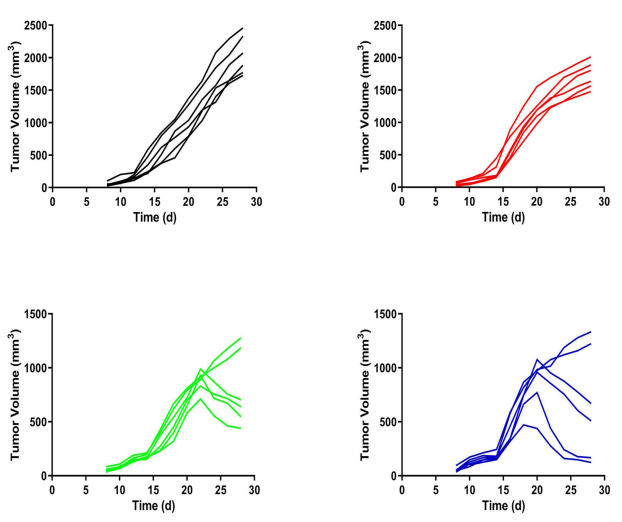

D

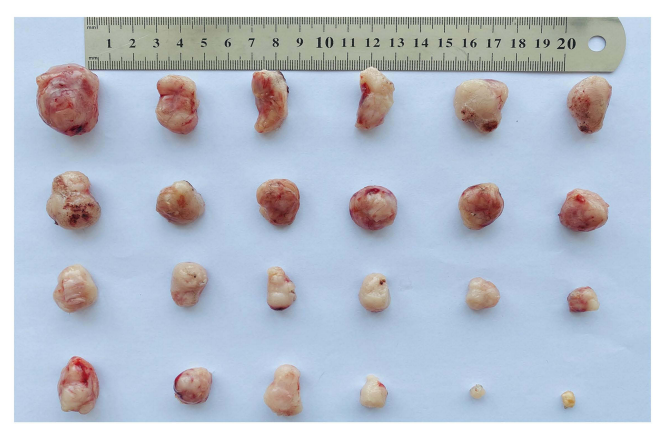

C

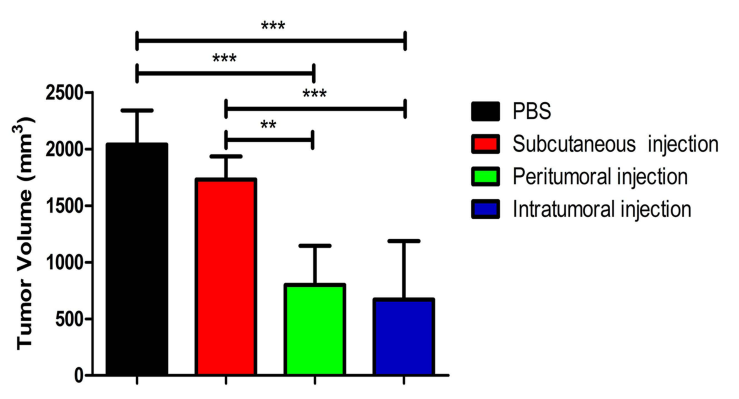

$\mathbf{E}$

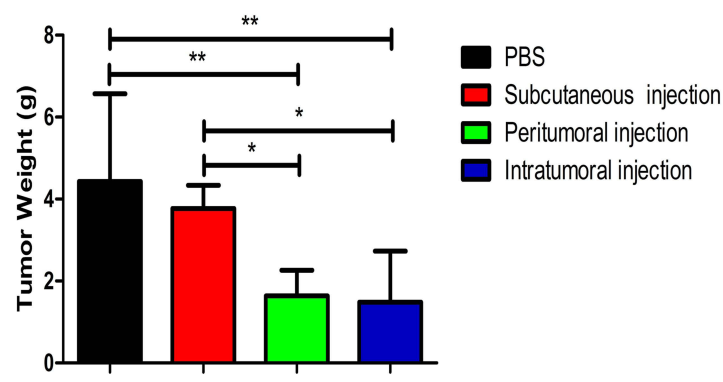

$\mathbf{F}$

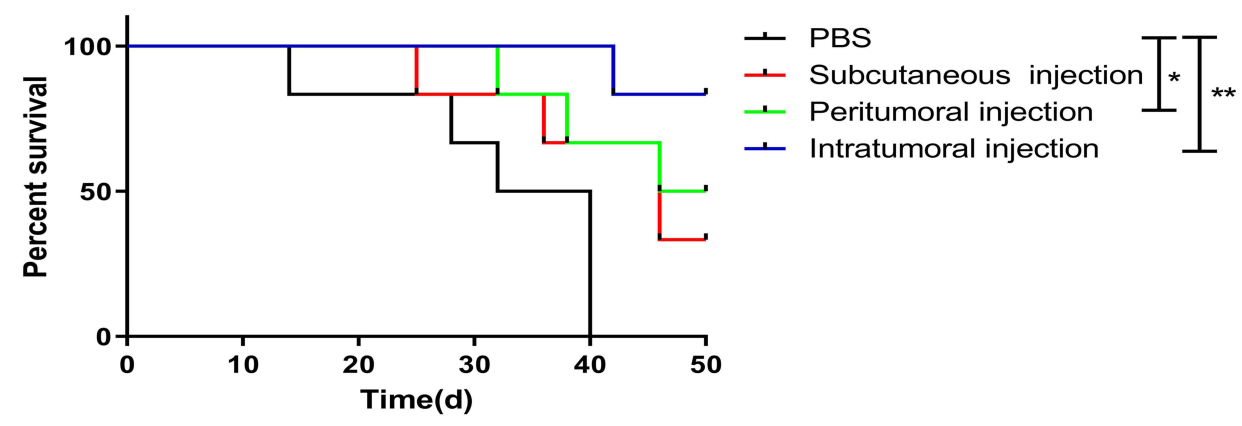

Figure I Effects of three different administration routes of vaccine on the inhibition of tumor growth. (A) Treatment schedule: All the mice were injected subcutaneously into the right flank with a single-cell suspension of $5 \times 10^{5} \mathrm{TC}-\mathrm{I}$ cells for tumor formation at day 0 . After tumor cell inoculation, tumor-bearing mice were treated with the vaccine by subcutaneous injection, peritumoral injection or intratumoral injection at days 9, 16, and 23. (B) Tumor volumes in the four groups were assessed over time. (C) Graphical representation of tumor volumes at day 28. (D) Photography of tumors excised from the mice at the endpoint of the experiment. From top to bottom, PBStreated group, subcutaneous injection group, peritumoral injection group, and intratumoral injection group. (E) Tumor weights (grams) at the time of excision. (F) KaplanMeier survival curves of different groups of tumor-bearing mice. The experiments were repeated twice and one of the two experiment results was presented. The data are expressed as the mean $\pm S D(n=6)$. The significance of the tumor volumes and the tumor weights was evaluated by one-way ANOVA followed by Tukey's multiple comparison test. Overall survival was estimated according to Kaplan-Meier methods and was compared using the Log rank test $\left({ }^{*} p<0.05\right.$, **p $<0.01$, *** $\left.p<0.00 \mathrm{I}\right)$. 
mouse spleens were harvested and dissociated to assess the proportion of immune cells by flow cytometry analysis. The proportion of CD4+ cells among total splenocytes was significantly increased from $8.03 \%$ in the control group to $12.38 \%(\mathrm{p}<0.001)$ in the subcutaneous injection group and $11.92 \% \quad(\mathrm{p}<0.01)$ in the intratumoral injection group (Figure 2A and B). The three different administration routes of the vaccine significantly improved the percentage of splenic CD8+ $\mathrm{T}$ cells (Figure 2C and D; control group: $3.67 \% \pm 3.12 \%$; subcutaneous group: $9.09 \% \pm 2.04 \%$, $\mathrm{p}<0.001$; peritumoral group: $8.55 \% \pm 0.68 \%, \mathrm{p}<0.01$; and intratumoral group: $8.16 \% \pm 0.61 \%, \mathrm{p}<0.01)$. The subcutaneous and intratumoral injection of the vaccine further enhanced IFN- $\gamma$ expression by 1.5 -fold $(\mathrm{p}<0.05)$ and 2 -fold $(\mathrm{p}<0.01)$ compared with that in the PBS control group (Figure 2E and F), respectively. Although there were significant differences in the percentage of IFN- $\gamma$-secreting cells between the four groups, the IFN- $\gamma$-secreting cell counts were low which might be attributed to the presence of dead cells in the analyzed cells. We next evaluated the impact of different administration routes of the vaccine on splenic immunosuppression. We found that subcutaneous $(p<0.05)$ and intratumoral $(p<0.01)$ treatment significantly decreased the proportion of splenic myeloid-derived suppressor cells (MDSCs) compared with the control group (Figure 2G and H). Furthermore, the data indicated that the three vaccination routes significantly reduced the percentage of splenic regulatory $\mathrm{T}$ cells (Tregs) and type 2-polarized tumor-associated macrophages (M2-TAMs). We observed a decreased fraction of CD25+Foxp3+ T cells within CD4 + cells in the subcutaneous $(15.90 \% \pm 1.24 \%, \mathrm{p}<0.05)$, peritumoral $(14.57 \% \pm 1.18 \%, \mathrm{p}<0.001)$, and intratumoral treatment group $(13.90 \% \pm 0.73 \%, \mathrm{p}<0.001)$ relative to the control group (17.93\% $\pm 1.44 \%$ ) (Figure $2 \mathrm{I}$ and J). The subcutaneous, peritumoral and intratumoral injection of the vaccine led to a decrease in F4/80+CD206+ cells among the CD11b+ cell population, resulting in a $32.83 \%, 44.91 \%$, and $63.65 \%$ decrease of M2-TAMs than that of control group, respectively (Figure $2 \mathrm{~K}$ and $\mathrm{L}$ ).

\section{Effects of Different Administration Routes of the Vaccine on Tumor-Infiltrating Lymphocytes}

To characterize the effects of the different administration routes of vaccine on the tumor-infiltrating lymphocytes (TILs), tumors were harvested 5 days after last treatment and analyzed by flow cytometry to allow a clear comparison of relative numbers of immune cells among treatment groups. The intratumoral injection of the vaccine increased the proportion of CD4+ T cells within the TILs $(11.49 \%$ $\pm 2.58 \%)$ compared with the control group $(7.59 \% \pm 1.38 \%$, $\mathrm{p}<0.05)$ and the subcutaneous injection group $(7.79 \%$ $\pm 2.47 \%, \mathrm{p}<0.05$ ) (Figure $3 \mathrm{~A}$ and $\mathrm{B}$ ). The peritumoral injection and intratumoral injection of the vaccine led to a significant increase in the density of CD8+ T cells, from $4.01 \%$ in the control group to $6.61 \%(\mathrm{p}<0.05)$ in the peritumoral injection group and $7.57 \%(\mathrm{p}<0.05)$ in the intratumoral injection group (Figure $3 \mathrm{C}$ and $\mathrm{D}$ ). The expression of IFN- $\gamma$ increased in all three treatment groups relative to the control group, albeit the differences were not significant (Figure $3 \mathrm{E}$ and $\mathrm{F}$ ). In addition, the intratumoral injection of the vaccine significantly reduced the percentage of MDSCs (CD11b+Gr-1+) from $11.39 \%$ in the control group to $6.76 \% \quad(p<0.05)$ (Figure $3 \mathrm{G}$ and $\mathrm{H})$. We observed a decreased fraction of CD25+Foxp3+ T cells among CD4 + cells in the intratumoral treatment group $(15.10 \% \pm 3.95 \%)$ relative to the control group $(23.33 \% \pm 1.26 \%, \mathrm{p}<0.05)$ and the subcutaneous injection group $(22.97 \% \pm 4.76 \%, \mathrm{p}<0.05)$ (Figure 3I and J). The intratumoral injection of the vaccine led to a decrease in F4/80+CD206+ cells within the CD11b+ cell population, resulting in a $33.49 \%, 30.31 \%$, and $27.03 \%$ decrease of M2-TAMs compared to the control group, subcutaneous, and peritumoral treatment group, respectively (Figure $3 \mathrm{~K}$ and $\mathrm{L}$ ). These results indicated that the intratumoral injection of the vaccine might promote the recruitment and activation of CD4+ and CD8+ T cells, reducing immunosuppressive cells including Tregs, MDSCs, and M2TAMs, demonstrating a reversal of the immunosuppressive phenotype of the TILs.

\section{Effects of Different Administration Routes of the Vaccine on Cytokines, Chemokines, and Matrix} Metalloproteinases (MMPs) in the Tumor

The level of transcripts of interleukin (IL)-2, IL-12, tumor necrosis factor alpha (TNF- $\alpha$ ), IFN- $\gamma$ were significantly increased by 5 -fold $(\mathrm{p}<0.001) \quad$ (Figure 4A), 3-fold $(p<0.001)$ (Figure $4 C), 2$-fold $(p<0.001)$ (Figure 4D), and 18 -fold $(\mathrm{p}<0.001)$ (Figure $4 \mathrm{~F})$, respectively, compared to those in the control group. The mRNA level of IL-10, transforming growth factor beta (TGF- $\beta$ ), CXCchemokine ligand (CXCL) 1, and vascular endothelial growth factor (VEGF) in the intratumoral group was significantly reduced by $47.60 \% \quad(p<0.01)$ (Figure 4B), 
A

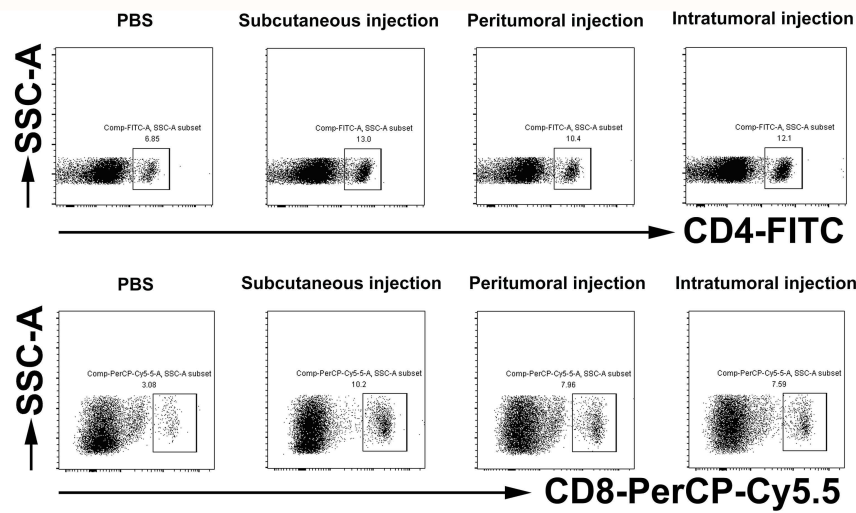

E

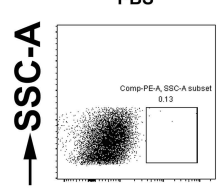

Subcutaneous injectio
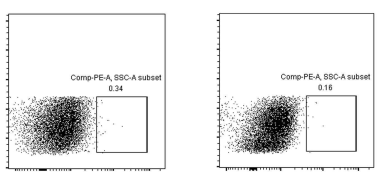

G

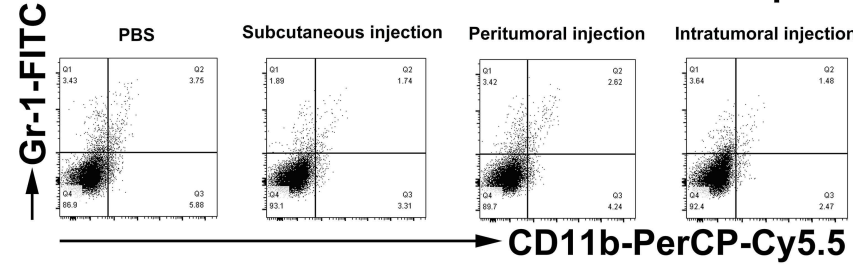

I

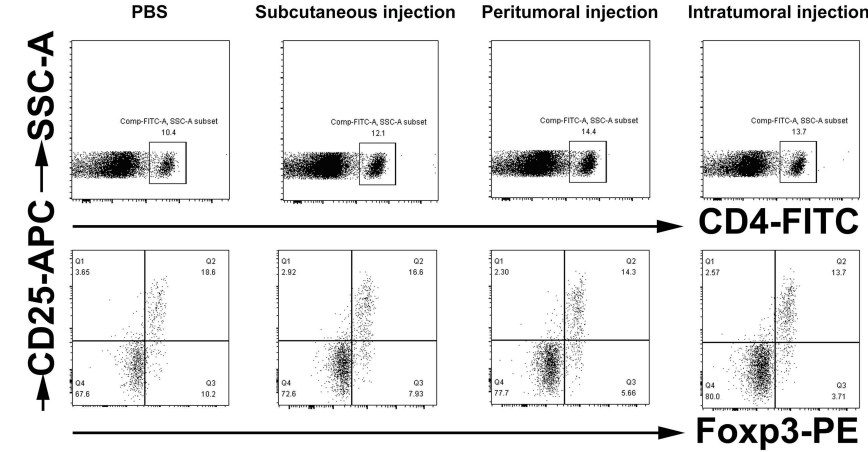

K

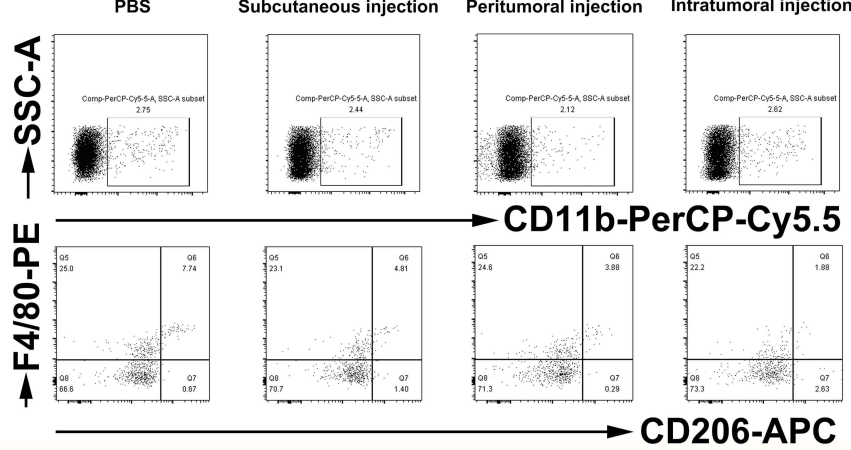

B

PBS

$\square$ Subcutaneous injection

$\square$ Peritumoral injection

Intratumoral injection

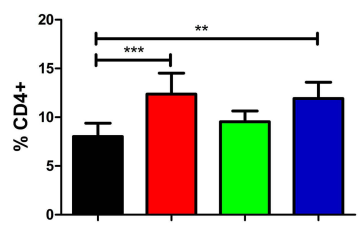

D

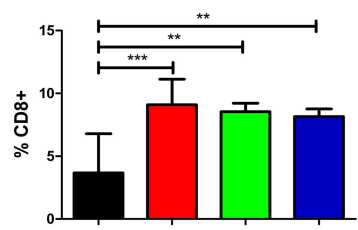

F

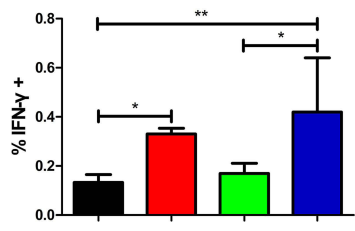

H
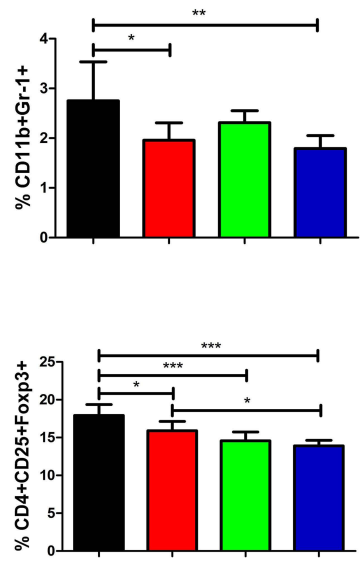

$\mathbf{L}$

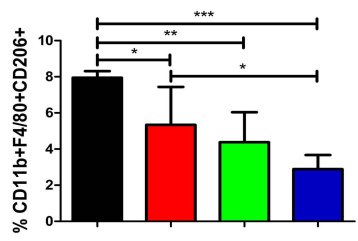

Figure 2 Effects of three different administration routes of the vaccine on the systemic immune responses. Representative cytometric dot plots of $(\mathbf{A}) \mathrm{CD} 4$, (C) CD8, (E) IFN- $\gamma$, (G) MDSCs, (I) Tregs, and (K) M2-TAMs. Flow cytometry data showing splenic CD4, CD8, and IFN- $\gamma$ positive cells represented as a bar graph expressed as (B) percentage of CD4+ T cells; (D) percentage of $\mathrm{CD} 8+$, and $(\mathbf{F})$ percentage of IFN- $\gamma$ cells. $(\mathbf{H})$ The frequency of splenic MDSCs expressed as the percentage of CDIIb and Gr-I double positive cells. (J) The percentage of splenic Tregs represented as the frequency of CD25 and Foxp3 double-positive cells among CD4+ T cells. (L) The fraction of M2-TAMs expressed as the frequency of F4/80 and CD206 double-positive cells within CDIl $b+$ splenocytes. The data are expressed as the mean \pm SD ( $n=3$ ). The significance of the data was evaluated by one-way ANOVA followed by Tukey's multiple comparison test $\left({ }^{*} p<0.05,{ }^{* *} p<0.01, * * * p<0.001\right)$. 
A

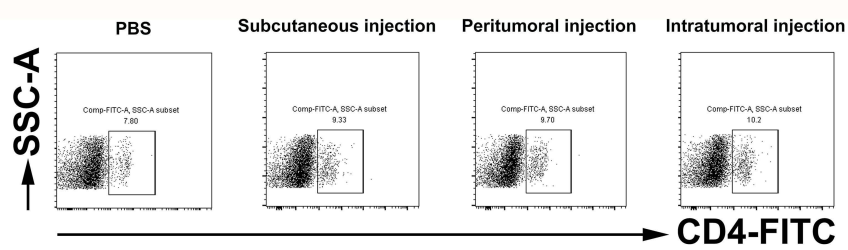

C

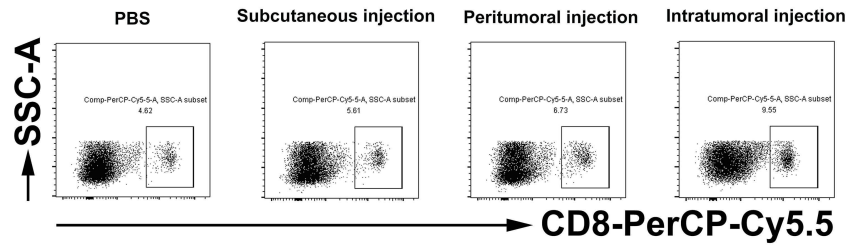

E

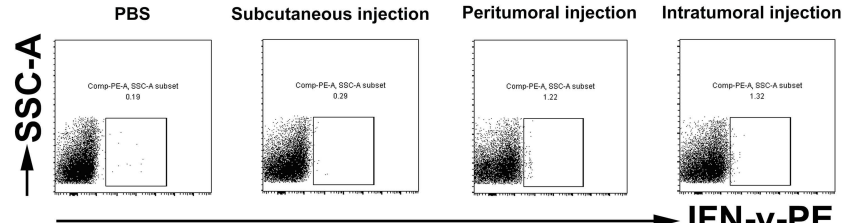

G

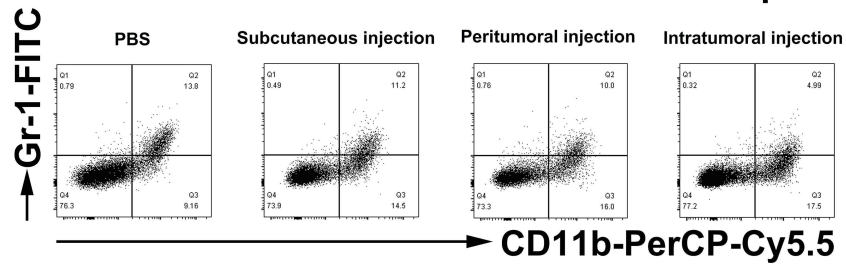

I

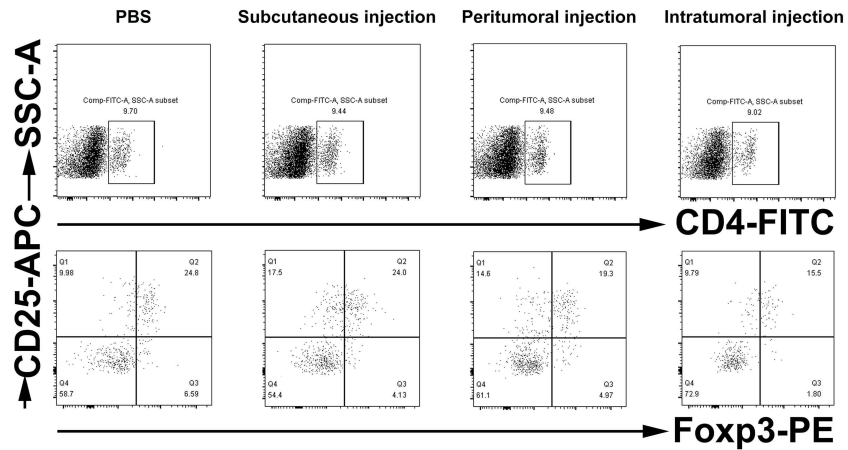

K

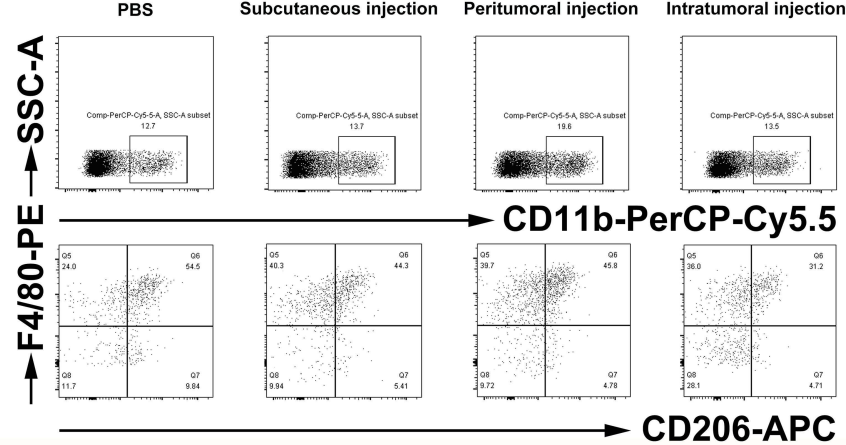

B

PBS

$\square$ Subcutaneous injection

$\square$ Peritumoral injection

Intratumoral injection

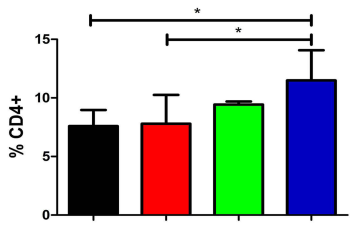

D

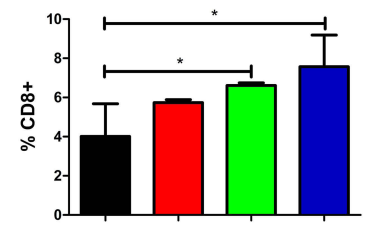

$\mathbf{F}$

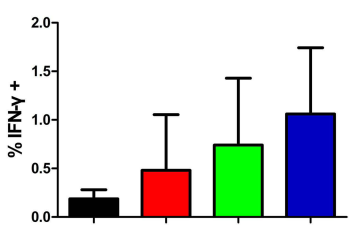

H

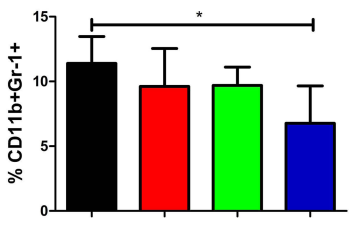

\section{J}

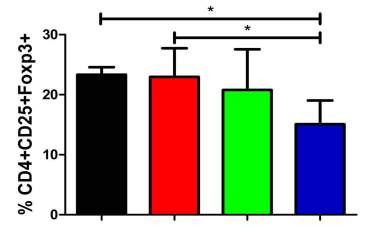

$\mathbf{L}$

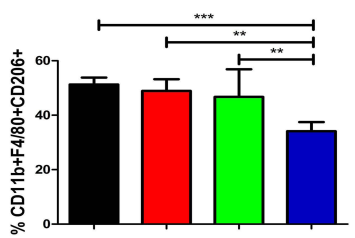

Figure 3 Effects of three different administration routes of the vaccine on TILs. The number of TILs was determined on day 28 by FACS. Representative flow cytometry results from one mouse of three mice showing tumor-infiltrating (A) CD4, (C) CD8, (E) IFN- $\gamma$, (G) MDSCs, (I) Tregs, and (K) M2-TAMs. Flow cytometry data showing intratumoral CD4, CD8 and IFN- $\gamma$ positive cells as represented as a bar graph expressed as (B) percentage of CD4+ T cells; (D) percentage of CD8+, and (F) percentage of IFN- $\gamma$ cells. (H) The frequency of tumor infiltrating MDSCs expressed as the percentage of CDI l b and Gr-I double-positive cells. (J) The percentage of intratumoral Tregs represented as the frequency of CD25 and Foxp3 double-positive cells among CD4+ T cells. (L) The fraction of M2-TAMs was expressed as the frequency of F4/80 and CD206 double positive cells within CDI l b+ tumor infiltrating cells. The data are expressed as the mean $\pm S D(n=3)$. The significance of the data was evaluated by one-way ANOVA followed by Tukey's multiple comparison test $\left({ }^{*} p<0.05,{ }^{* *} p<0.01, * * * p<0.00 I\right)$. 


\section{PBS $\square$ Subcutaneous injection $\square$ Peritumoral injection $\square$ Intratumoral injection}

A

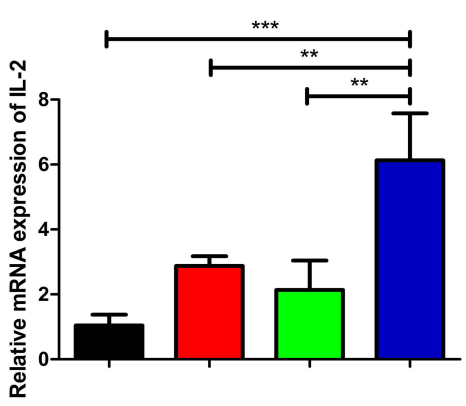

D

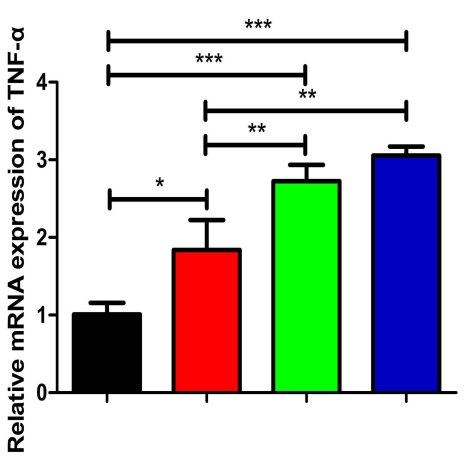

G

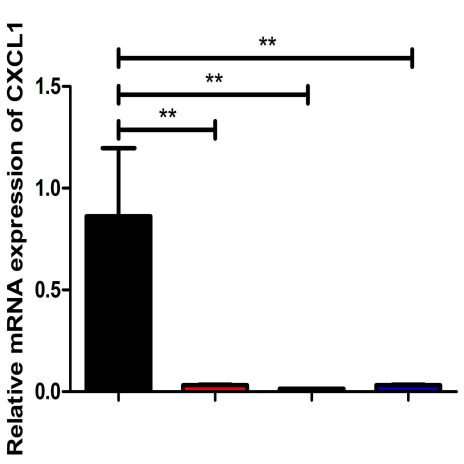

$\mathbf{J}$

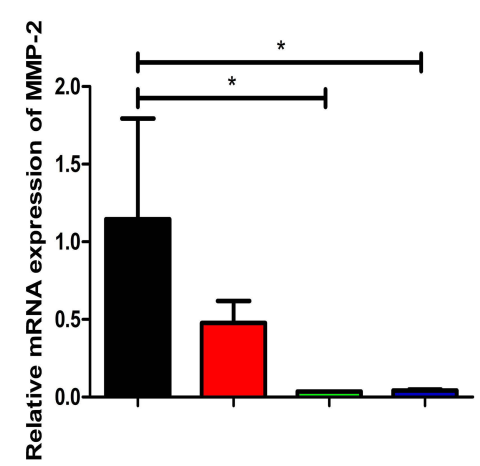

B 웅

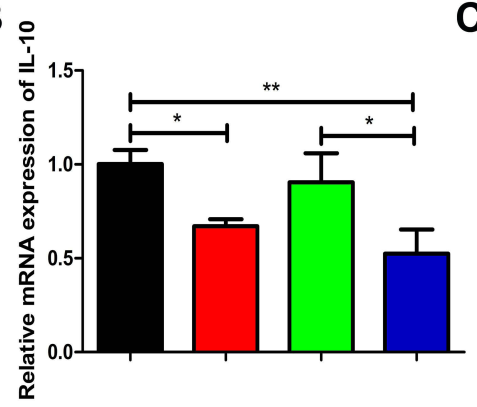

E

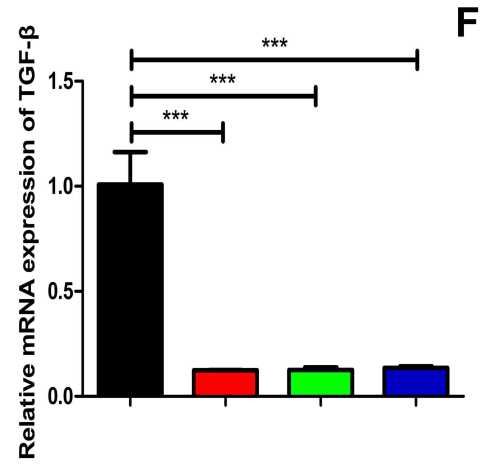

H
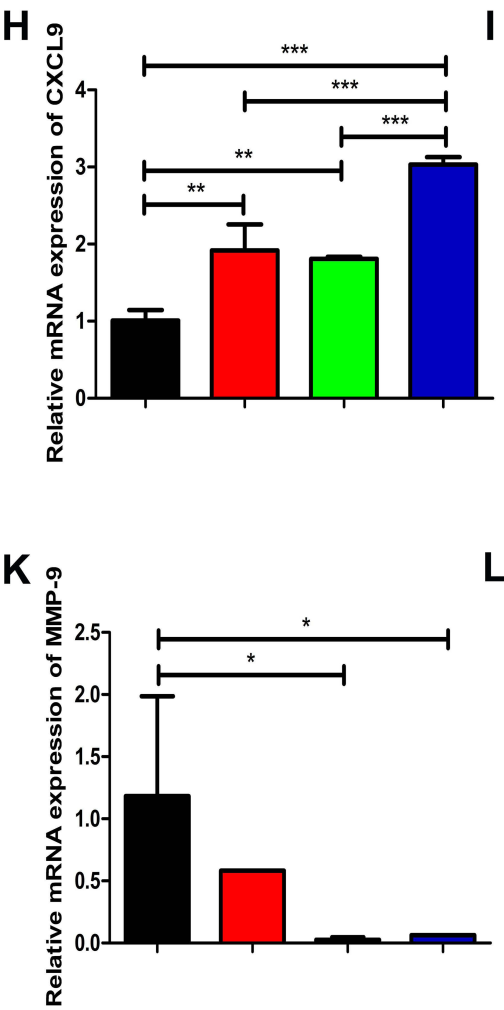

$\mathbf{F}$

L
C
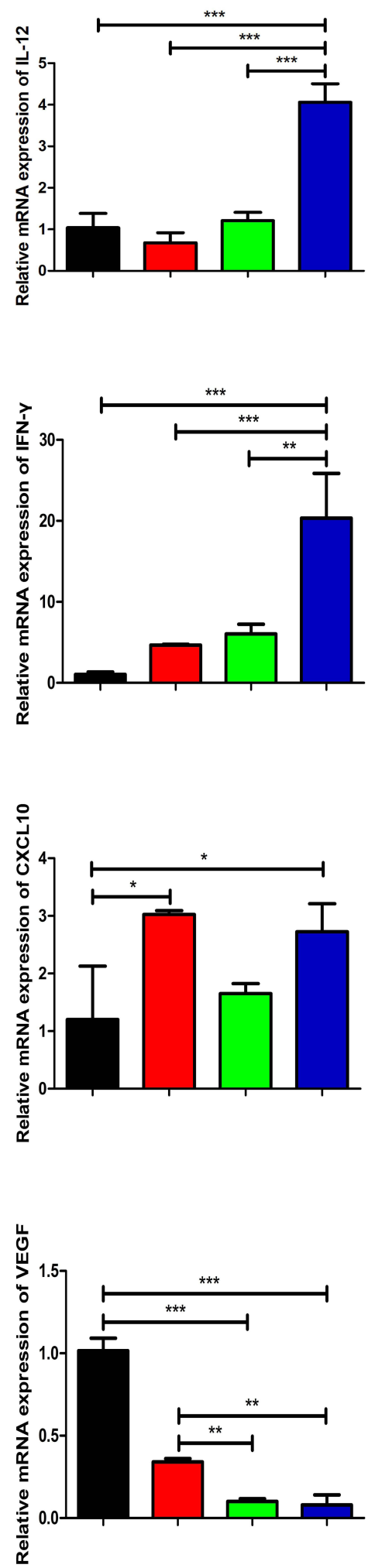

Figure 4 The relative mRNA expression of cytokines, chemokines and MMPs. The relative mRNA expression levels of (A) IL-2, (B) IL-I0, (C) IL-I2, (D) TNF- $\alpha$, (E) TGF- $\beta$, (F) IFN- $\gamma$, (G) CXCLI, (H) CXCL9, (I) CXCLI0, (J) MMP-2, (K) MMP-9, and (L) VEGF in the tumor tissue are shown. All PCR data were calculated relative to $\beta$-actin and represent the average $\pm S D$ of triplicate samples. The significance of the data was evaluated by one-way ANOVA followed by Tukey's multiple comparison test $\left({ }^{*} \mathrm{p}<0.05\right.$, $* * p<0.01, * * * p<0.001)$. 
$86.59 \% \quad(p<0.001) \quad($ Figure $\quad 4 \mathrm{E}), \quad 96.26 \% \quad(\mathrm{p}<0.01)$ (Figure 4G) and 92.14\% $(\mathrm{p}<0.001)$ (Figure $4 \mathrm{~L})$, respectively, compared to that in the control group. CXCL9 and CXCL10 mRNA expression levels in the tumor tissue of intratumoral injected mice were increased by 2-fold $(\mathrm{p}<0.001)$ (Figure $4 \mathrm{H})$ and 1 -fold $(\mathrm{p}<0.05)$ (Figure $4 \mathrm{I}$ ), respectively, compared to those of control mice. In addition to cytokines and chemokines, we also determined the expression levels of MMPs. The relative mRNA levels of MMP-2 and MMP-9 in the tumor tissue of intratumoral vaccinated mice were reduced by $96.24 \% \quad(\mathrm{p}<0.05)$ (Figure $4 \mathrm{~J}$ ) and $94.68 \%(\mathrm{p}<0.05)$ (Figure $4 \mathrm{~K}$ ), respectively, compared to those of control mice. Furthermore, the subcutaneous injection of the vaccine resulted in a significant increase in the relative expression of intratumoral TNF- $\alpha(\mathrm{p}<0.05)$ (Figure 4D), CXCL9 $(\mathrm{p}<0.01)$ (Figure $4 \mathrm{H}$ ), and CXCL10 $(\mathrm{p}<0.05)$ (Figure $4 \mathrm{I}$ ) and significantly decreased expression of intratumoral IL-10 $(p<0.05)$ (Figure 4B), TGF- $\beta(p<0.001)$ (Figure 4E), and CXCL1 $(p<0.01)$ (Figure $4 \mathrm{G}$ ) compared to that of control mice. Likewise, the relative expression of TGF- $\beta$ $(p<0.001)$ (Figure 4E), CXCL1 $(p<0.01)$ (Figure 4G), MMP-2 $\quad(p<0.05) \quad($ Figure $\quad 4 J), \quad$ MMP-9 $\quad(p<0.05)$ (Figure 4K), and VEGF $(p<0.001)$ (Figure $4 \mathrm{~L}$ ) in peritumoral vaccination mice significantly decreased compared to that of the control group. In addition, the peritumoral injection of the vaccine significantly increased the level of intratumoral TNF- $\alpha(p<0.001)$ (Figure 4D) and CXCL9 $(p<0.01)$ (Figure $4 \mathrm{H})$.

\section{Effects of Different Administration Routes of the Vaccine on CD4, CD8, CD3I, and $\alpha$-SMA Expression}

The representative immunohistochemical staining in Figure $5 \mathrm{~A}$ and $\mathrm{B}$ indicated the expression of $\mathrm{CD} 4+$ and $\mathrm{CD} 8+$ cells in tumor tissue. The tumor infiltrated CD4+ and CD8+ T lymphocytes were distributed around both the tumor tissue and the blood vessels. The peritumoral injection and intratumoral injection of the vaccine significantly increased the density of $\mathrm{CD} 4+$ and $\mathrm{CD} 8+\mathrm{T}$ cells relative to the control group (Figure 5E and F). The intratumoral microvessel density (MVD) was quantified by measuring CD31-positive staining which was mainly detected in the vascular endothelial cell membrane. The IHC images suggested that neoplastic endothelial cells forming the vascular structure were positive and other regions of the tumor showed low expression of CD31 (Figure 5C). All capillaries expressing membranous CD31 were counted at $400 \times$ magnification. We found a significantly less MVD in the intratumoral group (1.91 \pm 0.30$)$ than in the control group $(3.09 \pm 0.83)$ and the subcutaneous group (2.91 \pm 1.30$)$ (Figure $5 \mathrm{G}$ ), which suggested that the intratumoral injection of the vaccine significantly reduced the MVD in the tumors. $\alpha$-SMA positive staining was mainly detected in the tumor stroma, the IHC staining results indicated that $\alpha$-SMA expression in the intratumoral injection group was significantly lower than that in the control group (Figure 5D and $\mathrm{H}$ ).

\section{Discussion}

In this study, we explored the effects of three routes of vaccination on the inhibition of TC- 1 grafted tumors. We found that intratumoral and peritumoral vaccination reversed tumor growth when compared to subcutaneously vaccination or compared to control treatment (Figure 1B-E). Considering the results of our previous studies in which the antitumor effect of subcutaneous vaccination was demonstrated for early-stage tumors, ${ }^{6,7}$ we speculated that subcutaneous injection may be more effective for early-stage tumors. For late-stage tumors, subcutaneous inoculation might not significantly attenuate the growth rate of tumors (Figure 1B-E). Although peritumoral injection inhibited tumor growth, the intratumoral injection of the vaccine elicited a stronger antitumor effect, with two of the six mice becoming nearly tumor-free at day 28 (Figure 1B and D). Furthermore, the antitumor effect translated into improved survival in the intratumoral injected mice $(\mathrm{p}<0.01)$ as compared to the untreated mice (Figure 1F). In short, we observed significant tumor regression and survival improvement when the vaccine was delivered directly into the tumor tissue as compared to either untreated or subcutaneous injection of the vaccine.

Ishida et $\mathrm{al}^{16}$ and Peng et $\mathrm{al}^{17}$ recently demonstrated superior effects of intratumoral delivery of HPV16 E7 peptide-based vaccines using TC-1 tumor model in C57BL/6 mice; however, there are several differences between these studies and the present study. First, the formulation of the vaccine in our study and in the two similar studies differed. The vaccine used in the study by Ishida et al ${ }^{16}$ was a HPV 16 E7 49-57 short peptide, which contained only a single CD8 epitope, and Peng et $\mathrm{al}^{17}$ used two short peptides as antigens, which were HPV 16 E7 49-57 peptide containing a CD8 epitope and the HPV 16 E7 43-62 peptide containing a CD4 epitope. In our current study, we used the HPV 16 E7 43-77 long peptide vaccine, which not only contains a CD8 epitope, but also 
A

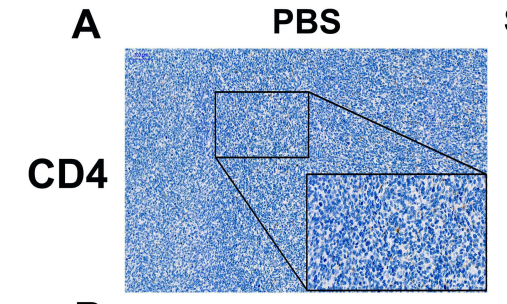

B
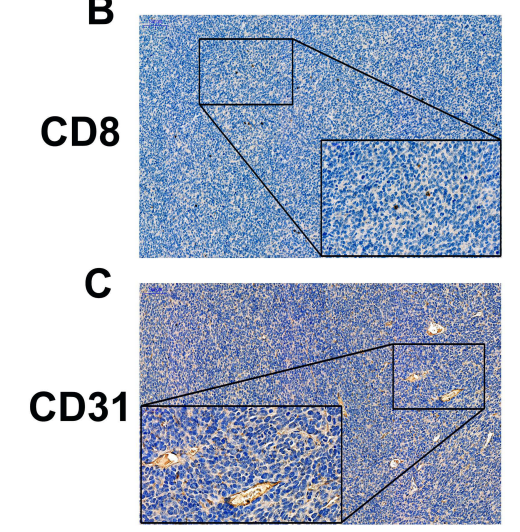

D

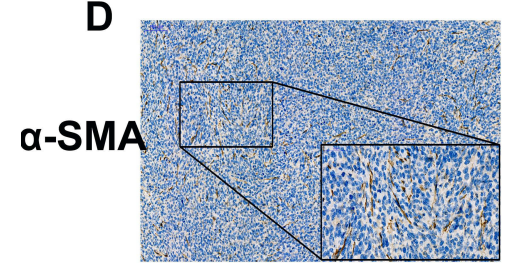

E
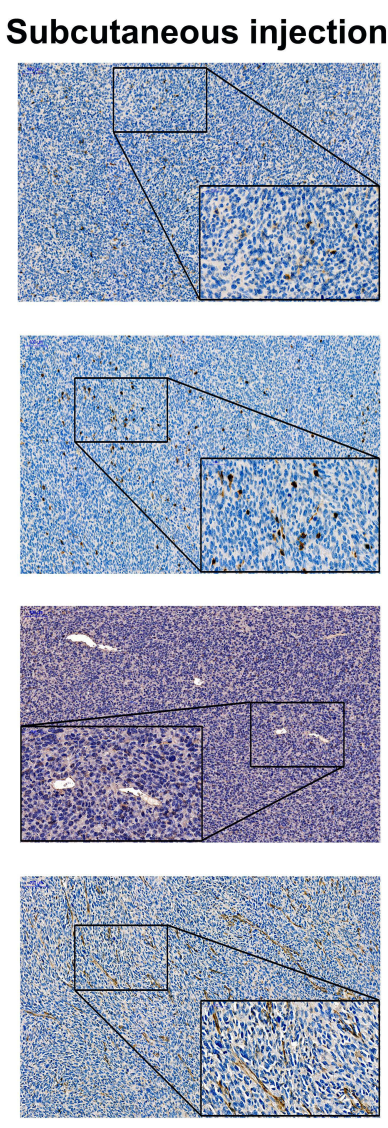
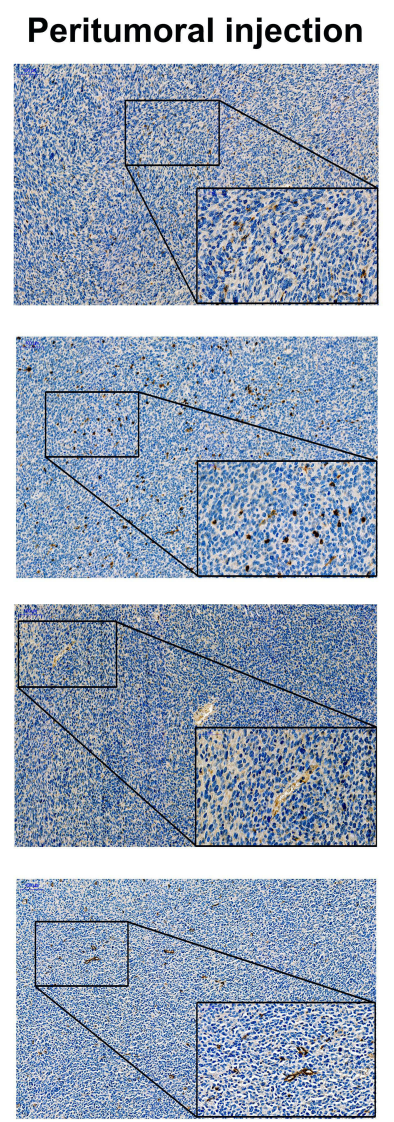

$\mathbf{F}$
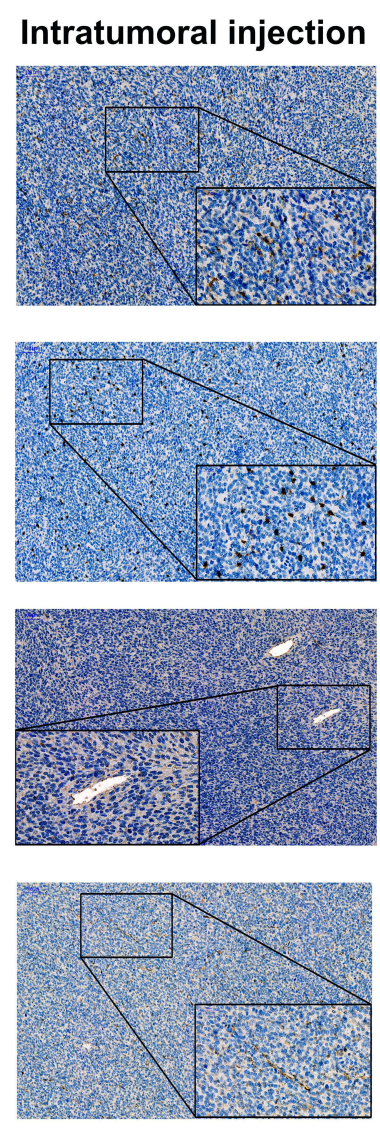

\section{$\square$ PBS $\square$ Subcutaneous injection $\square$ Peritumoral injection $\square$ Intratumoral injection}
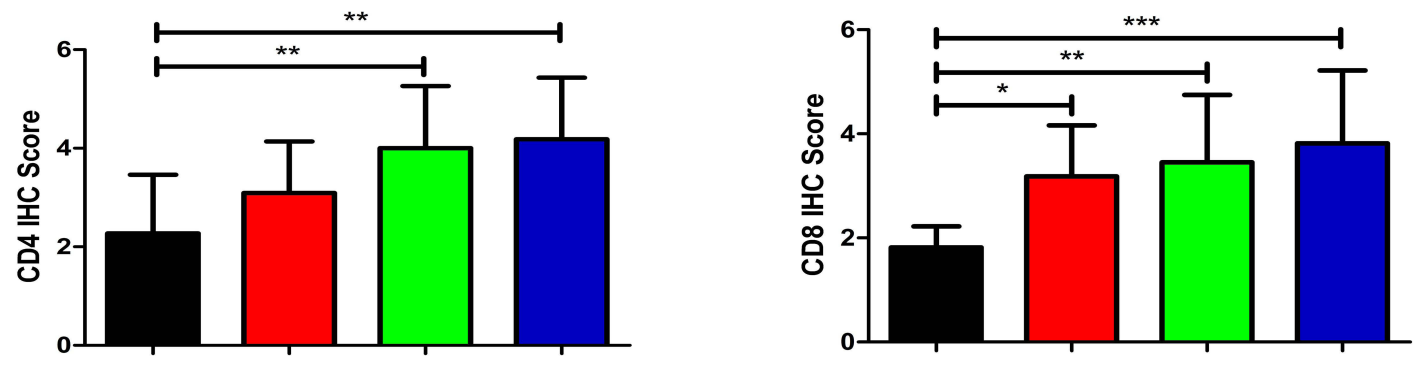

G

H
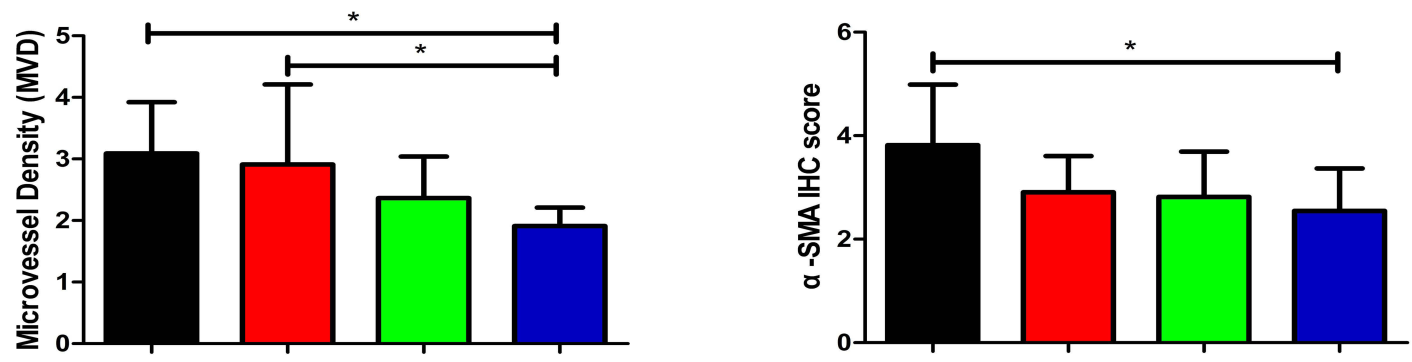

Figure 5 The abundance of CD4, CD8, CD31, and $\alpha$-SMA expression by immunohistochemistry analysis. Representative images of (A) CD4, (B) CD8, (C) CD3I, and (D) $\alpha$-SMA staining are shown. Immunohistochemistry score of (E) CD4, (F) CD8, and (H) $\alpha$-SMA. (G) Intratumoral MVD was quantified by measuring CD3I-positive staining. The scale bar is located in the upper left corner of the immunohistochemistry image. Images are shown at $100 \times$ magnification and scale bars $=100 \mu \mathrm{m}$. The insert images are at $400 \times$ magnification and scale bars $=50 \mu \mathrm{m}$. The data are expressed as the mean \pm SD. The significance of the data was evaluated by one-way ANOVA followed by Tukey's multiple comparison test $(* \mathrm{p}<0.05, * * \mathrm{p}<0.01$, $* * * \mathrm{p}<0.001)$. 
contains two CD4 epitopes. CD4 cells play a vital role in the anti-tumor immune response. Activated Th1 CD4 cells are capable of producing IFN- $\gamma$ which mediates the enhancement of MHC expression on tumor cells, the recognition and eradication of tumor cells by $\mathrm{T}$ cells. ${ }^{22}$ Furthermore, CD4 T cells are required for infiltration of CD8 T cells to the tumor site, ${ }^{23-25}$ which is critical for the efficacy of immunotherapy and for long-term antitumor effects. In addition, the adjuvant used in Ishida et al's study was the TLR3 agonist poly (I:C), while the adjuvant used in our study was the TLR9 agonist CpG ODN. We believe that the efficient inoculation route for vaccines with different formulations may differ. Differences in adjuvants will also have an impact on the anti-tumor effects of therapeutic vaccines. It is worth investigating effective inoculation routes for given vaccine formulations. Moreover, Ishida et $\mathrm{al}^{16}$ administered the vaccine when the tumor xenograft volume in mice was about $50 \mathrm{~mm}^{3}$, while the vaccine intervention was also performed during the early stage of tumor formation (at days 3, 6, and 10) in the study by Peng et al. ${ }^{17}$ Our previous research results demonstrated that the HPV E7 43-77 long peptide vaccine with $\mathrm{CpG}$ ODN as an adjuvant in the early stage of tumor formation in mice (Day 4, tumor volume was approximately $50 \mathrm{~mm}^{3}$ ) showed excellent tumor inhibition effects. Tumor dimensions of mice were significantly reduced, and some tumors even disappeared. ${ }^{6}$ But as the results of our current study have shown, when the tumor volume in mice reached a certain level (eg, $150 \pm 50 \mathrm{~mm}^{3}$ ), the subcutaneous administrated vaccines cannot completely control tumor growth, which may be due to the potential presence of an immunosuppressive TME. Thus, we evaluated the intratumoral or peritumoral routes of vaccination, and evaluated the impact of different vaccination routes on the TME. Last but not least, cervical cancer has an insidious onset, and most cervical cancer patients found in the clinic are middle to late stage patients in low- and middle-income countries. $^{26,27}$ We believe that exploring the therapeutic effect of vaccines on larger or advanced tumors has greater clinical value.

Therapeutic cancer vaccines aim to promote immune responses, and in particular, activate cytotoxic $\mathrm{CD} 8+$ $\mathrm{T}$ cells that are specific to tumor antigens. ${ }^{28}$ In order to better understand the mechanisms underlying differences in antitumor effects, we examined the effects of the different administration routes of the vaccine on systemic and tumor local immune responses. We found that subcutaneous injection of the vaccine induced stronger systemic immune responses, but the local immune responses were weaker than that of intratumoral and peritumoral vaccine routes, which further demonstrated that the local immune response played a major role in the antitumor effect. Subcutaneous injections activate dermal dendritic cells and Langerhans cells, promoting an effective antigen processing and eventually effectively stimulating cellular immune response and humoral immune responses, ${ }^{29,30}$ which might explain why subcutaneous vaccination induced the significant systemic immune response. Instead, the intratumoral injection of the vaccine enhanced the locoregional antitumor efficacy by enabling high bioavailability of vaccines directly at the injected tumor sites and reduced systemic toxicity. ${ }^{31}$ In addition, the flow cytometry results of tumor cells showed that only CD8+ T cells in the peritumoral injection group increased significantly, but the antitumor effect of the peritumoral group was adequate. We hypothesized that the presence of CD8+ T cells in the TME are crucial to the rejection of the tumor induced by the vaccine. However, we have not performed knockout studies to verify these hypotheses at present, but this will be addressed in future studies. Essentially, our results suggested that intratumoral injection of the vaccine triggered not only local immunologic responses but also systemic immunologic responses, both of which contributed to an improved antitumor response.

The bottleneck for cancer immunotherapy is the immunosuppressive TME in which the tumor cells are located. ${ }^{32}$ MDSCs, Tregs, M2-TAMs, and inhibitory cytokines are the major components of the immune system that cause the subversion of antitumor immunity in the TME. ${ }^{32}$ MDSCs elicit immunosuppression systemically and locally in the TME through different pathways, including promoting cancer cell proliferation and survival, angiogenesis and lymph angiogenesis, and the expression of immunosuppressive cytokines. ${ }^{33}$ Tregs are potent inhibitors of the immune system, suppressing $\mathrm{T}$ cells, B cells, natural killer (NK) cells, dendritic cells, and macrophages via humoral and cell-cell contact mechanisms. In the majority of solid tumors in the cervix, kidney, breast, and melanomas, the high frequency of tumor-infiltrating Tregs is often associated with poor prognosis. ${ }^{34}$ Macrophages are polarized into two distinct phenotypes, the classically activated macrophages (M1-TAMs), which are pro-inflammatory, pro-immunity, and antitumor, or the alternative activated macrophages (M2-TAMs), which are anti-inflammatory, 
immune suppressive, and pro-tumor. ${ }^{35}$ M2-TAMs promote tumor growth and metastases through a variety of diverse mechanisms, including facilitating angiogenesis, matrix breakdown, and tumor-cell motility. ${ }^{36}$ Thus, we also focused our attention on the effects of different vaccination routes on systemic and tumor local immunosuppressive cells. We found decreased splenic MDSCs in subcutaneous and intratumoral group (Figure $2 \mathrm{G}$ and $\mathrm{H}$ ), as well as reduced splenic Tregs and M2-TAMs in all the three vaccinated groups (Figure 2I-L), while only intratumoral vaccination significantly reduced the frequency of tumor-infiltration MDSCs, Tregs, and M2-TAMs (Figure 3G-L). We hypothesized that the low frequency of tumor-infiltration MDSCs, Tregs and M2-TAMs in the intratumoral injection group might be associated with relieving immunosuppression and reinstating antitumor immune responses.

To better understand how intratumoral vaccination can significantly enhance the generation of tumor-infiltrating CD4+ and CD8+ $\mathrm{T}$ cells and the antitumor response, we assessed transcriptional changes of cytokines, chemokines, and MMPs in the tumor. IL-2 plays an important role in the development of adaptive immune response as it mediates the proliferation of $\mathrm{T}$ cells and differentiation into effector lymphocytes. ${ }^{37}$ IL-12 is released by a variety of immune cells upon infection or inflammation to induce differentiation of Th1 cells and increase activity of CTLs as well as IFN- $\gamma$ production. ${ }^{38}$ Studies have indicated that TNF- $\alpha$ is frequently used in tumor immunotherapy while IFN- $\gamma$ can directly inhibit tumor cell proliferation. ${ }^{39}$ CXCL9 and CXCL10 promote the migration of effector $\mathrm{T}$ cells and NK cells into tumors. ${ }^{40}$ Our experimental results demonstrated that compared with untreated controls, the three vaccination routes tested herein, increased the expression level of IL-2 (Figure 4A), IL-12 (Figure 4C), TNF- $\alpha$ (Figure 4D), IFN- $\gamma$ (Figure 4F), CXCL9 (Figure 4H), and CXCL10 (Figure 4I) in the tumor to varying degrees, but the intratumoral injection group showed the largest increase and the strongest effect. The increased numbers of tumor-infiltrating effector $\mathrm{T}$ cells in the intratumoral group might be associated with increased levels of antitumor cytokines as well as CXCL9 and CXCL10 chemokines. MDSCs, ${ }^{41}$ Tregs, ${ }^{42}$ and $\mathrm{M} 2-\mathrm{TAMs}^{43}$ block $\mathrm{T}$ cell immune responses specific to tumor antigens via the production of immunosuppressive molecules including TGF- $\beta$, IL-10. TGF- $\beta$ directly inhibits the stimulation, differentiation, proliferation, and effector function of CD4+ and CD8+ T cells that mediate immune responses. ${ }^{44}$ IL-10, a known suppressive cytokine of T-cell proliferation and cytokine production, markedly reduces the function of effector $\mathrm{T}$ cells. ${ }^{45}$ Studies have shown that the ablation of CXCL1 in tumor cells increased $\mathrm{CD} 8+\mathrm{T}$ cell infiltration and induced responses to immunotherapy. ${ }^{46}$ Our qRT-PCR results have shown that the intratumoral injection of the vaccine induced a significant decrease in IL-10 (Figure 4B), TGF- $\beta$ (Figure 4E) and CXCL1 (Figure 4G) levels compared to those of control mice. We supposed that the significant increase of the pro-inflammatory and antitumor cytokines, and the decrease of these immunosuppressive cytokines in the intratumoral injection group can promote antitumor immunity and enhance the efficacy of immunotherapy.

HPV-related cancer such as cervical cancer is a spectrum disease with a gradual progression from pre-malignant condition to aggressive disease, which begins in the cervix, and later spreads towards the lower uterine segment, vagina, paracervical space, along with the broad and uterosacral ligaments. $^{47}$ Among patients with cervical cancer, approximately $4.16 \%-7.7 \%$ develop lung metastasis, $0.8 \%-23 \%$ develop bone metastases, and the overall outcome of patients with metastatic cervical cancer is poor. ${ }^{48}$ Thus, we evaluated indicators related to cervical cancer invasion and metastasis, including MMP-2, MMP-9, and $\alpha$-SMA and their correlated expression with the different administration route of the vaccine. MMP-2 may enhance the invasive ability in cervical tumors by facilitating basement membrane and extracellular matrix degradation. ${ }^{49}$ MMP-9 is an early marker of tumor progression in squamous lesions of the cervix, and MMP-9 mRNA expression is up-regulated in tumor and stromal cells of both high-grade cervical intraepithelial neoplasia and invasive squamous cell carcinoma of the cervix. ${ }^{50}$ The level of MMP-2 and MMP-9 mRNA in the subcutaneous injection group was similar to those of a previous study, which was about $50 \%$ lower than that of the untreated control group. ${ }^{7}$ The level of these MMPs mRNA in the intratumoral injection group was significantly reduced by approximately $90 \%$ compared to the control group (Figure 4J and K). Cancer-associated fibroblasts are major components of the surrounding stroma of cervical carcinomas, which may display an altered phenotype compared with normal fibroblasts characterized by increased expression of $\alpha$-SMA and fibroblast activation protein. ${ }^{51}$ The lowest expression of $\alpha$-SMA in the intratumoral injection group was demonstrated in Figure 5D and H. Overall, our results showed marked downregulation of MMP-2, MMP-9, and $\alpha$-SMA in the intratumoral vaccination group which might contribute to the suppression of tumor invasion and metastasis. 
Angiogenesis plays an important role in high-grade cervical intraepithelial neoplasia and invasive squamous carcinoma of the cervix, as in other types of pre-malignant lesions and carcinoma; ${ }^{52}$ thus, we detected markers of angiogenesis in tumors. A number of studies have shown that the HPV oncoprotein E6 might contribute to cervical cancer angiogenesis by direct stimulation of the VEGF gene, and the level of VEGF expression was associated with poor prognosis and early recurrence in cervical cancer. ${ }^{53-55}$ In addition, cervical cancer angiogenesis measured by CD31 MVD is an independent prognostic factor for both progression-free survival and overall survival in high-risk, early-stage cervical cancer patients. ${ }^{56}$ In this current study, we found that the reduced level of VEGF mRNA (Figure 4L) and the decreased average MVD (Figure 5G) in the tumor tissue of mice in the intratumoral injection group, which indicated that intratumoral injection of the vaccine could significantly inhibit the formation of tumor microvessels by regulating the expression and transcription levels of angiogenesis-related factors.

In the present study, we investigated the differences and mechanisms of antitumor effects induced by different vaccination routes, but the mechanisms underlying the different immune responses were not fully investigated and will be the focus of future studies. In our follow-up experiments, we will establish a multi-tumor model in mice to evaluate whether the intratumoral injection of the vaccine in a given tumor will also lead to the elimination of distant lesions. Further, we will deplete $\mathrm{CD} 8+\mathrm{T}$ cells using neutralizing antibodies in mice to verify that $\mathrm{CD} 8+\mathrm{T}$ cells are crucial to the antitumor effect induced by the vaccine.

\section{Conclusion}

We explored the anti-TC-1-grafted tumors activity induced by three different administration routes of an HPV therapeutic vaccine containing HPV 16 E7 43-77 peptide in combination with the toll-like receptor-9 agonist $\mathrm{CpG}$ ODN. Our results suggested that the intratumoral vaccination route generated an enhanced antitumor effect relative to subcutaneous vaccination route by improving the TME, and may have a significant impact on developing efficacious therapeutic vaccines for HPV-associated diseases including cervical cancer.

\section{Abbreviations}

HPV, human papillomavirus; CpG ODN, unmethylated cytosine-phosphate-guanosine oligodeoxynucleotide; MDSCs, myeloid-derived suppressor cells; Tregs, regulatory T cells;
M2-TAMs, type 2-polarized tumor-associated macrophages; MMPs, matrix metalloproteinases; CTL, cytotoxic T lymphocyte; qRT-PCR, quantitative real-time reverse transcription polymerase chain reaction; CXCL, CXCchemokine ligand; ANOVA, analysis of variance; MVD, microvessel density; NK, natural killer; Th1, T-helper type 1; SD, standard deviation; TILs, tumor-infiltrating lymphocytes; $\alpha$-SMA, alpha-smooth muscle actin; VEGF, vascular endothelial growth factor; IL, interleukin; TNF- $\alpha$, tumor necrosis factor alpha; TGF- $\beta$, transforming growth factor beta; IFN- $\gamma$, interferon gamma.

\section{Funding}

This work was supported by the National Natural Science Foundation of China (No. 81472439).

\section{Disclosure}

The authors report no conflicts of interest in this work.

\section{References}

1. Crosbie EJ, Einstein MH, Franceschi S, Kitchener HC. Human papillomavirus and cervical cancer. Lancet. 2013;382 (9895):889-899. doi:10.1016/S0140-6736(13)60022-7

2. Monie A, Hung CF, Roden R, Wu TC. Cervarix: a vaccine for the prevention of HPV 16, 18-associated cervical cancer. Biologics. 2008;2(1):97-105.

3. Clark KT, Trimble CL. Current status of therapeutic HPV vaccines. Gynecol Oncol. 2020;156(2):503-510. doi:10.1016/j.ygyno.2019.12.017

4. Hoppe-Seyler K, Bossler F, Braun JA, Herrmann AL, Hoppe-Seyler F. The HPV E6/E7 oncogenes: key factors for viral carcinogenesis and therapeutic targets. Trends Microbiol. 2018;26(2):158-168. doi:10.1016/j.tim.2017.07.007

5. Nayereh KG, Khadem G. Preventive and therapeutic vaccines against human papillomaviruses associated cervical cancers. Iran J Basic Med Sci. 2012;15(1):585-601.

6. Yang Y, Che Y, Zhao Y, Wang X. Prevention and treatment of cervical cancer by a single administration of human papillomavirus peptide vaccine with $\mathrm{CpG}$ oligodeoxynucleotides as an adjuvant in vivo. Int Immunopharmacol. 2019;69:279-288. doi:10.1016/j. intimp.2019.01.024

7. Che Y, Yang Y, Suo J, An Y, Wang X. Induction of systemic immune responses and reversion of immunosuppression in the tumor microenvironment by a therapeutic vaccine for cervical cancer. Cancer Immunol Immunother. 2020;69(12):2651-2664. doi:10.1007/s00262-020-02651-3

8. Hammerich L, Bhardwaj N, Kohrt HE, Brody JD. In situ vaccination for the treatment of cancer. Immunotherapy. 2016;8(3):315-330. doi:10.2217/imt. 15.120

9. Burn Aschner C, Pierce C, Knipe DM, Herold BC. Vaccination route as a determinant of protective antibody responses against herpes simplex virus. Vaccines. 2020;8:2.

10. Marabelle A, Tselikas L, de Baere T, Houot R. Intratumoral immunotherapy: using the tumor as the remedy. Ann Oncol. 2017;28 (suppl_12):xii33-xii43. doi:10.1093/annonc/mdx683

11. Newman JH, Chesson CB, Herzog NL, et al. Intratumoral injection of the seasonal flu shot converts immunologically cold tumors to hot and serves as an immunotherapy for cancer. Proc Natl Acad Sci U S A. 2020;117(2):1119-1128. doi:10.1073/pnas.1904022116 
12. Lee JM, Lee MH, Garon E, et al. Phase I Trial of Intratumoral Injection of CCL21 gene-modified dendritic cells in lung cancer elicits tumor-specific immune responses and CD8(+) T-cell Infiltration. Clin Cancer Res. 2017;23(16):4556-4568. doi:10.1158/ 1078-0432.CCR-16-2821

13. Ursu R, Carpentier A, Metellus $P$, et al. Intracerebral injection of $\mathrm{CpG}$ oligonucleotide for patients with de novo glioblastoma-A Phase II multicentric, randomised study. Eur J Cancer. 2017;73:30-37. doi:10.1016/j.ejca.2016.12.003

14. Lee SY, Kang TH, Knoff J, et al. Intratumoral injection of therapeutic HPV vaccinia vaccine following cisplatin enhances HPV-specific antitumor effects. Cancer Immunol Immunother. 2013;62 (7):1175-1185. doi:10.1007/s00262-013-1421-y

15. Nichols AJ, Gonzalez A, Clark ES, et al. Combined systemic and intratumoral administration of human papillomavirus vaccine to treat multiple cutaneous basaloid squamous cell carcinomas. JAMA Dermatol. 2018;154(8):927-930. doi:10.1001/ jamadermatol.2018.1748

16. Ishida E, Lee J, Campbell JS, et al. Intratumoral delivery of an HPV vaccine elicits a broad anti-tumor immune response that translates into a potent anti-tumor effect in a preclinical murine HPV model. Cancer Immunol Immunother. 2019;68(8):1273-1286. doi:10.1007/ s00262-019-02357-1

17. Peng S, Tan M, Li YD, et al. PD-1 blockade synergizes with intratumoral vaccination of a therapeutic HPV protein vaccine and elicits regression of tumor in a preclinical model. Cancer Immunol Immunother. 2020;70:1049.

18. Yang MC, Yang A, Qiu J, et al. Buccal injection of synthetic HPV long peptide vaccine induces local and systemic antigen-specific CD8 + T-cell immune responses and antitumor effects without adjuvant. Cell Biosci. 2016;6:17. doi:10.1186/s13578-016-0083-9

19. Kawarada Y, Ganss R, Garbi N, Sacher T, Arnold B, Hammerling GJ. $\mathrm{NK}$ - and $\mathrm{CD} 8(+) \mathrm{T}$ cell-mediated eradication of established tumors by peritumoral injection of $\mathrm{CpG}$-containing oligodeoxynucleotides. J Immunol. 2001;167(9):5247-5253. doi:10.4049/jimmunol.16 7.9.5247

20. Ono T, Harada M, Yamada A, et al. Antitumor effects of systemic and local immunization with a CTL-directed peptide in combination with a local injection of OK-432. Clin Cancer Res. 2006;12 (4):1325-1332. doi:10.1158/1078-0432.CCR-05-1293

21. Brianti P, De Flammineis E, Mercuri SR. Review of HPV-related diseases and cancers. New Microbiol. 2017;40(2):80-85.

22. Romagnani S. T-cell subsets (Th1 versus Th2). Ann Allergy Asthma Immunol. 2000;85(1):9-18. doi:10.1016/S1081-1206(10)62426-X

23. Bos R, Sherman LA. CD4+ T-cell help in the tumor milieu is required for recruitment and cytolytic function of $\mathrm{CD} 8+\mathrm{T}$ lymphocytes. Cancer Res. 2010;70(21):8368-8377. doi:10.1158/ 0008-5472.CAN-10-1322

24. Marzo AL, Kinnear BF, Lake RA, et al. Tumor-specific CD4+ T cells have a major "post-licensing" role in CTL mediated anti-tumor immunity. $J$ Immunol. 2000;165(11):6047-6055. doi:10.4049/ jimmunol.165.11.6047

25. Wong SB, Bos R, Sherman LA. Tumor-specific CD4+ T cells render the tumor environment permissive for infiltration by low-avidity CD8 $+\mathrm{T}$ cells. J Immunol. 2008;180(5):3122-3131. doi:10.4049/ jimmunol.180.5.3122

26. Mesafint Z, Berhane Y, Desalegn D. Health seeking behavior of patients diagnosed with cervical cancer in Addis Ababa, Ethiopia. Ethiop J Health Sci. 2018;28(2):111-116. doi:10.4314/ejhs.v28i2.2

27. Mwaka AD, Okello ES, Wabinga H, Walter FM. Symptomatic presentation with cervical cancer in Uganda: a qualitative study assessing the pathways to diagnosis in a low-income country. $B M C$ Womens Health. 2015;15(1):15. doi:10.1186/s12905-015-0167-4

28. Butterfield LH. Cancer vaccines. BMJ. 2015;350:h988. doi:10.1136/ bmj.h988
29. Romani N, Thurnher M, Idoyaga J, Steinman RM, Flacher V. Targeting of antigens to skin dendritic cells: possibilities to enhance vaccine efficacy. Immunol Cell Biol. 2010;88(4):424-430. doi:10.1038/icb.2010.39

30. Nicolas JF, Guy B. Intradermal, epidermal and transcutaneous vaccination: from immunology to clinical practice. Expert Rev Vaccines. 2008;7(8):1201-1214. doi:10.1586/14760584.7.8.1201

31. Hamid O, Ismail R, Puzanov I. Intratumoral Immunotherapy-Update 2019. Oncologist. 2020;25(3):e423-e438. doi:10.1634/theoncologist.2019-0438

32. Kalathil SG, Thanavala Y. High immunosuppressive burden in cancer patients: a major hurdle for cancer immunotherapy. Cancer Immunol Immunother. 2016;65(7):813-819. doi:10.1007/ s00262-016-1810-0

33. Sica A, Massarotti M. Myeloid suppressor cells in cancer and autoimmunity. $J$ Autoimmun. 2017;85:117-125. doi:10.1016/j. jaut.2017.07.010

34. Tanaka A, Sakaguchi S. Regulatory T cells in cancer immunotherapy. Cell Res. 2017;27(1):109-118. doi:10.1038/cr.2016.151

35. Brown JM, Recht L, Strober S. The Promise of Targeting Macrophages in Cancer Therapy. Clin Cancer Res. 2017;23 (13):3241-3250. doi:10.1158/1078-0432.CCR-16-3122

36. Pollard JW. Tumour-educated macrophages promote tumour progression and metastasis. Nat Rev Cancer. 2004;4(1):71-78. doi:10.1038/ nrc 1256

37. Spolski R, Li P, Leonard WJ. Biology and regulation of IL-2: from molecular mechanisms to human therapy. Nat Rev Immunol. 2018;18 (10):648-659. doi:10.1038/s41577-018-0046-y

38. Zundler S, Neurath MF. Interleukin-12: functional activities and implications for disease. Cytokine Growth Factor Rev. 2015;26 (5):559-568. doi:10.1016/j.cytogfr.2015.07.003

39. Kearney CJ, Vervoort SJ, Hogg SJ, et al. Tumor immune evasion arises through loss of TNF sensitivity. Sci Immunol. 2018;3:23. doi:10.1126/sciimmunol.aar3451

40. Nagarsheth N, Wicha MS, Zou W. Chemokines in the cancer microenvironment and their relevance in cancer immunotherapy. Nat Rev Immunol. 2017;17(9):559-572. doi:10.1038/nri.2017.49

41. Marvel D, Gabrilovich DI. Myeloid-derived suppressor cells in the tumor microenvironment: expect the unexpected. J Clin Invest. 2015;125(9):3356-3364. doi:10.1172/JCI80005

42. Schmidt A, Oberle N, Krammer PH. Molecular mechanisms of treg-mediated $\mathrm{T}$ cell suppression. Front Immunol. 2012;3:51. doi:10.3389/fimmu.2012.00051

43. Sawa-Wejksza K, Kandefer-Szerszen M. Tumor-associated macrophages as target for antitumor therapy. Arch Immunol Ther Exp (Warsz). 2018;66(2):97-111. doi:10.1007/s00005-017-0480-8

44. Sheng J, Chen W, Zhu HJ. The immune suppressive function of transforming growth factor-beta (TGF-beta) in human diseases. Growth Factors. 2015;33(2):92-101. doi:10.3109/ 08977194.2015.1010645

45. Taylor A, Verhagen J, Blaser K, Akdis M, Akdis CA. Mechanisms of immune suppression by interleukin-10 and transforming growth factor-beta: the role of $\mathrm{T}$ regulatory cells. Immunology. 2006;117 (4):433-442. doi:10.1111/j.1365-2567.2006.02321.x

46. Li J, Byrne KT, Yan F, et al. Tumor cell-intrinsic factors underlie heterogeneity of immune cell infiltration and response to immunotherapy. Immunity. 2018;49(1):178-193 e177. doi:10.1016/j. immuni.2018.06.006

47. Hassan RN, Luo H, Jiang W. Effects of nicotinamide on cervical cancer-derived fibroblasts: evidence for therapeutic potential. Cancer Manag Res. 2020;12:1089-1100. doi:10.2147/CMAR. S229395

48. Li H, Wu X, Cheng X. Advances in diagnosis and treatment of metastatic cervical cancer. J Gynecol Oncol. 2016;27(4):e43. doi:10.3802/jgo.2016.27.e43 
49. Davidson B, Goldberg I, Kopolovic J, et al. MMP-2 and TIMP-2 expression correlates with poor prognosis in cervical carcinoma-a clinicopathologic study using immunohistochemistry and mRNA in situ hybridization. Gynecol Oncol. 1999;73(3):372-382. doi:10.1006/gyno.1999.5381

50. Davidson B, Goldberg I, Kopolovic J, et al. Expression of matrix metalloproteinase-9 in squamous cell carcinoma of the uterine cervix-clinicopathologic study using immunohistochemistry and mRNA in situ hybridization. Gynecol Oncol. 1999;72(3):380-386. doi:10.1006/gyno.1998.5285

51. Erdogan B, Webb DJ. Cancer-associated fibroblasts modulate growth factor signaling and extracellular matrix remodeling to regulate tumor metastasis. Biochem Soc Trans. 2017;45(1):229-236. doi:10.1042/ BST20160387

52. Tomao F, Papa A, Rossi L, et al. Angiogenesis and antiangiogenic agents in cervical cancer. Onco Targets Ther. 2014;7:2237-2248. doi:10.2147/OTT.S68286
53. Lopez-Ocejo O, Viloria-Petit A, Bequet-Romero M, Mukhopadhyay D, Rak J, Kerbel RS. Oncogenes and tumor angiogenesis: the HPV-16 E6 oncoprotein activates the vascular endothelial growth factor (VEGF) gene promoter in a p53 independent manner. Oncogene. 2000;19 (40):4611-4620. doi:10.1038/sj.onc. 1203817

54. Fujimoto J, Toyoki H, Sato E, Sakaguchi H, Tamaya T. Clinical implication of expression of vascular endothelial growth factor-C in metastatic lymph nodes of uterine cervical cancers. $\mathrm{Br} J$ Cancer. 2004;91(3):466-469. doi:10.1038/sj.bjc.6601963

55. Choi CH, Song SY, Choi JJ, et al. Prognostic significance of VEGF expression in patients with bulky cervical carcinoma undergoing neoadjuvant chemotherapy. BMC Cancer. 2008;8:295. doi:10.1186/ 1471-2407-8-295

56. Randall LM, Monk BJ, Darcy KM, et al. Markers of angiogenesis in high-risk, early-stage cervical cancer: a Gynecologic Oncology Group study. Gynecol Oncol. 2009;112(3):583-589. doi:10.1016/j. ygyno.2008.11.013

\section{Publish your work in this journal}

Cancer Management and Research is an international, peer-reviewed open access journal focusing on cancer research and the optimal use of preventative and integrated treatment interventions to achieve improved outcomes, enhanced survival and quality of life for the cancer patient.
The manuscript management system is completely online and includes a very quick and fair peer-review system, which is all easy to use. Visit http://www.dovepress.com/testimonials.php to read real quotes from published authors. 